\title{
Life with a Microcalorimeter EDS Detector on a FEG-SEM
}

\author{
E.A. Kenik* and H. Demers**** \\ *Oak Ridge National Laboratory, Metals and Ceramics Division, Oak Ridge, TN, USA \\ McGill University, Dept. Mining, Metals and Materials Engineering, Montreal, Quebec, Canada
}

The addition of a microcalorimeter ( $\mu$ cal) EDS detector to a FEG-SEM capable of x-ray microanalysis at low accelerating voltages should provide the next improvement in high spatial and spectral resolution microanalysis of bulk materials [1,2]. The current generation of $\mu \mathrm{cal}$ EDS detectors features a spectral resolution $(<15 \mathrm{eV})$ that is $\sim 10 \mathrm{x}$ better than a conventional Si EDS detector, with higher peak-to-background, fewer peak overlaps and improved and detection limits in cases where peak overlap is a concern [3]. These improvements partially offset the loss of mediumenergy $x$-ray lines that are not excited under low voltage operation. Count rate limitations of the $\mu \mathrm{cal}$ detector on quantitative analysis and mapping have been examined.

The Low Voltage Microprobe (LVM) at Oak Ridge National Laboratory is based on a JEOL 6500F FEG-SEM capable of a high probe current $(>40 \mathrm{nA})$ in a small diameter $(<10 \mathrm{~nm})$ probe at low accelerating voltages $(\leq 5 \mathrm{kV})$. The LVM is equipped with the first commercially available $\mu$ cal EDS detector, manufactured by EDAX/Vericold, as well as a conventional Si(Li) EDS detector capable of high count rates for fast mapping applications. The complexities of the $\mu$ cal EDS detector and the differences in operation compared to a conventional Si(Li) EDS detector have been explored. Points examined include the effects of the multiple thermal shields/windows and ice formation, the thin film nature of the x-ray absorber, the implications of using an x-ray optic to increase collection efficiency and the energy resolution and count rate limitations of the detector. Though much of the day-to-day operation of the $\mu$ cal EDS analysis system is automated under software control, there are manual operations required for optimized pulse processing, calibration and analysis of spectra.

Materials applications of the LVM have been selected to highlight both the capabilities and current limitations of the $\mu \mathrm{cal}$ EDS detector. These applications include a variety of materials, ranging from pure elemental standards to complex, advanced materials such as superalloys, vanadium alloys, ODS ferritic alloys, solid oxide fuel cell materials and semiconductor device and multi-layered hard drive materials. These studies include both point analyses and elemental mapping.

Multi-layered materials, such as the magnetic recording media for a computer hard drive, offer challenges in specimen preparation for microanalysis. In addition, a diagnostic tool would be very helpful to follow and validate fabrication steps. Performing microanalysis in the SEM at a series of different accelerating voltages allows the depth distribution of elements to be determined and is the basis of such software packages as Strata for determining such depth distributions. Figure 1 shows several $\mu \mathrm{cal}$ EDS spectra from a hard disk at several voltages. At $10 \mathrm{kV}$ (1a), X-rays from the Co-Pt recording media as well as many of the others layers are observed. At $5 \mathrm{kV}(1 \mathrm{~b})$, the intensities of Xrays from the glass substrate and other deep layers decrease or disappear (e.g., Si, Na, K). At even lower voltages, X-ray generation is limited to the outer layers (lubricant, protection and media layers). The complicating effect of decreasing over-voltages must be taken into account, but from a series of such measurements the depth distribution of elements in a multi-layered or inhomogeneous material can be inferred. Making such measurements with a $\mu$ cal EDS detector permits the use of higher order (lower energy) x-ray lines with reduced chances of peak overlap.

Spectrum imaging with the $\mu \mathrm{cal}$ detector offers several attractive benefits in complex materials, including reduced peak overlap for closely spaced elemental lines and higher spatial resolution for mapping at lower voltages. Backscattered electron imaging and elemental mapping for a CMSX4 superalloy are shown in Figure 2. The element maps show that the darkly-imaging $\gamma^{\prime}$ phase is enriched in $\mathrm{Al}$ and Ta; whereas the brightly-imaging $\gamma$ phase is enriched in $\mathrm{Cr}$ and Re. Even though the Re M $\alpha$ peak strongly overlaps the W M $\beta$ peak (1842 and $1835 \mathrm{eV}$, respectively), the uniformity 
of the $\mathrm{W}$ distribution (Figure 1f) permits the strong segregation of Re to the $\gamma$ phase to be revealed. The presence of the lower intensity $\mathrm{W} \mathrm{M} \beta$ peak results in a constant background in Figure 2(f).

References

[1] D. A. Wollman, S. W. Nam, G. C. Hilton, K. D. Irwin, N. F. Bergren, D. A. Rudman, J. A. Martinis and D. E. Newbury, J. Microscopy 199 (2000) 37.

[2] D. A. Wollman, S. W. Nam, D. E. Newbury, G. C. Hilton, K. D. Irwin, N. F. Bergren, S. Deiker, D. A. Rudman and J. A. Martinis, Nucl. Instr. Meth. in Phys. Res. A 444 (2000) 145.

[3] D. Newbury, D. Wollman, K. Irwin, G. Hilton and J. Martinis, Ultramicroscopy 78 (1999) 73.

[4] Research at the Oak Ridge National Laboratory SHaRE User Facility was sponsored by the Division of Materials Sciences and Engineering (EAK, HD), U.S. Department of Energy, under contract DE-AC05-00OR22725 with UT-Battelle, LLC.
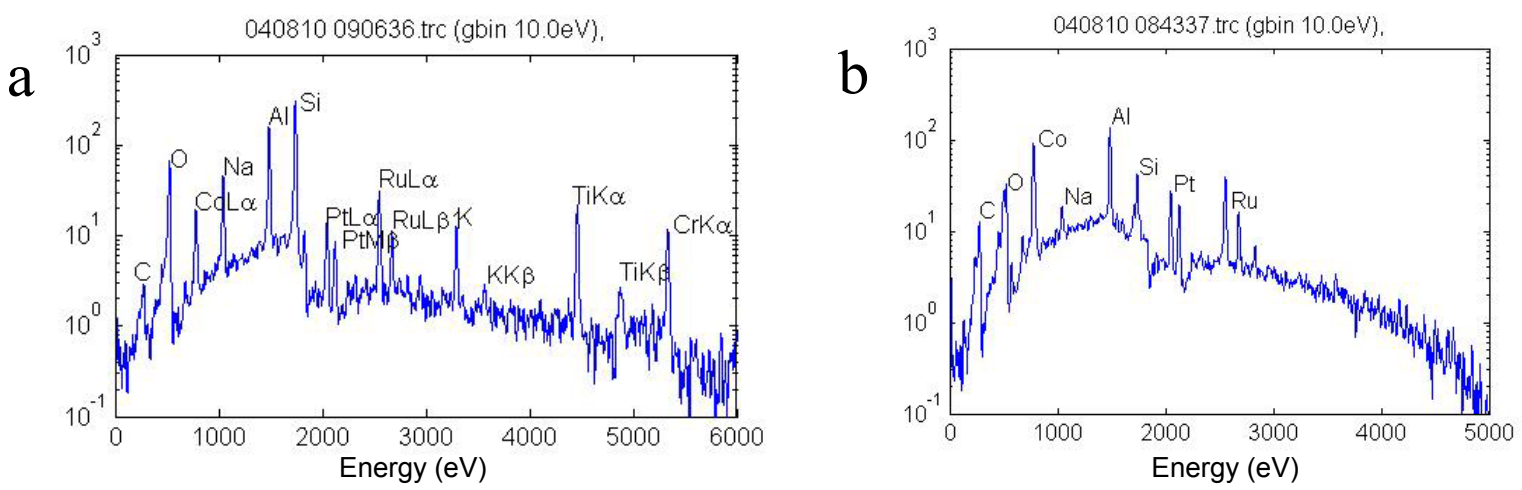

Figure 1 Microcalorimeter EDS spectra for magnetic recording media from a hard drive with accelerating voltages of (a) $10 \mathrm{kV}(\sim 0.3 \mathrm{nA}, \sim 95 \mathrm{cps})$ and (b) $5 \mathrm{kV}(\sim 0.4 \mathrm{nA}, \sim 90 \mathrm{cps})$ for 600 second acquisitions. Differences in peak intensities are related to depth distribution of the elements in the multiple layers on the glass substrate and changes in over-voltage. Note the relative intensity decreases for elements in the lower layers $(\mathrm{Si}, \mathrm{Na}, \mathrm{K})$ at $5 \mathrm{kV}$. The $\mathrm{Si}$ and $\mathrm{O} \mathrm{K}$ absorption edges are from the silica polycapillary x-ray optic.

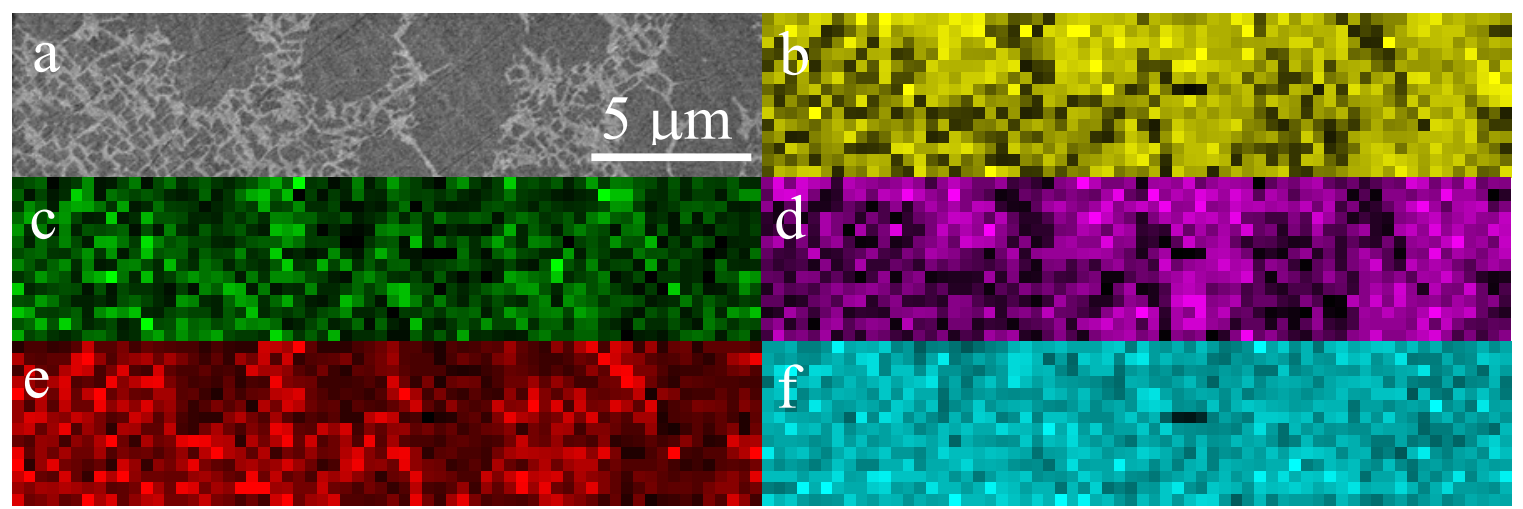

Figure 2 Low-voltage microscopy and microanalysis of CMSX4 superalloy: (a) BSE image and elemental maps of (b)Al, (c) Co, (d) Ta, (e) Re and (f) W, respectively. The dark phase in (a) is the ordered $\gamma^{\prime}$ phase and the bright phase is the disordered $\gamma$ phase. The spectrum image (64 x 14 pixels, $0.37 \mu \mathrm{m}$ spacing) was acquired at $5 \mathrm{kV}, 0.3 \mathrm{nA}, \sim 300 \mathrm{cps}$ throughput count rate and a 10 second per pixel dwell ( $\sim 2.5 \mathrm{~h}$ total acquisition time). 